\title{
Recovery and Purification of Glycerine as By-product from Philippine Coconut Methyl Ester
}

\author{
Annabelle V. Briones ${ }^{1, *}$ \\ ${ }^{1}$ Industrial Technology Development Institute, Department of Science and Technology, DOST Complex, Gen. Santos Ave., Bicutan, \\ Taguig City, Philippines 1631 \\ *Corresponding author: avbriones2003@yahoo.com
}

\author{
KEYWORDS \\ Coconut Methyl Ester \\ Glycerine \\ MONG (matter organic \\ nonglycerol) \\ Transesterification
}

\begin{abstract}
The study is about the recovery and purification of glycerine as a by-product from coconut methyl ester production in the Philippines. The aqueous layer produced from settling or phase splitting of the methyl ester after the transesterification process was subjected to various treatments like acidification, neutralization, concentration in vacuo in order to get back the crude glycerine. The crude glycerine obtained from the laboratory and scale-up process conformed with the specification set by the British standard for crude glycerine. The recovered glycerine is composed of: $84.92 \%$, glycerine; $8.03 \%$, ash; $4.72 \%, \mathrm{H} 20 ; 2.32 \%$, MONG. Further distillation yielded a refined glycerine that meets with the specification set by USP. The average glycerine content of refined glycerine is $96.86 \%$; ash, $0.06 \%$; water, $1.10 \%$, refractive index @ $20^{\circ} \mathrm{C}, 1.4696$, specific gravity at $25^{\circ} \mathrm{C}, 1.296 \mathrm{~g}$.
\end{abstract}

(c) The Author(s) 2019. This article is distributed under a Creative Commons Attribution-ShareAlike 4.0 International license.

\section{INTRODUCTION}

Glycerol is the main by-product of biodiesel production; about $10 \%$ of the weight of biodiesel is generated in glycerol. A large amount of glycerol generated may become an environmental problem since it cannot be disposed of in the environment (Quispe et al. 2013). In the Philippines, Coconut methyl ester (CME) is the main Philippine biodiesel feedstock and is an oleochemical derived from Coconut Oil (CNO), which is a product from crushing copra, the dried meat of the coconut (Purdy and Corpuz 2019). Due to the emergence of biofuels and the use of alternative energy, the supply of glycerine also increases. It is therefore imperative that a glycerine recovery and purification technology be developed and viable to small and medium scale processors. The objectives of the study were: 1 . To optimize processing conditions to produce the crude glycerine by various treatments; 2. Purify the recovered crude glycerine as a by-product in the production of methyl ester; 3 . To analyze and evaluate the physicochemical properties of the crude glycerine and refined glycerine.

According to the Department of Energy of the Philippines, there are 11 operational biodiesel refineries as of June 30, 2017 (registered with the Philippine Board of Investment) with an aggregate annual capacity of approximately 575 Million Liters. The need to recover and process the byproducts of coconut methyl ester (CME) is deemed necessary since this will help in increasing the profit of CME manufacturers and indirectly reduce the operating/production cost of CME. Aside from this aspect, it is also important to recycle these by-products considering that these will generate a voluminous amount of wastes and pose a bigger problem for disposal. Recycling of by-products also creates jobs to millions of unemployed Filipinos.
Glycerol is an important by-product of many important oleochemical processes and its value is a significant contributor to the economic viability of the oleochemical industry and its products (Gunstone and Heming 2004). The growing demand for fatty acids, fatty alcohols, and of esters for biodiesel is leading to increased production of glycerol (Gunstone and Heming 2004). The balance between glycerol supply versus demand and of the consequences of this on the oleochemical industry is a great concern (Gunstone and Heming 2004).

\section{MATERIALS AND METHODS}

The aqueous layer containing a mixture of glycerine, free fatty acids, water, inorganic salts, methyl esters, unreacted mono-, di-, and triglycerides and a variety of other matter organic non-glycerol (MONG) was collected from one of the biodiesel refineries located in Romblon, Philippines and from the pilot production of coconut methyl ester using the Chemicals and Energy Division of the Industrial Technology Development Institute facilities. All chemicals used were of technical grade except for the chemicals used for analysis of samples which are of analytical grade.

\subsection{Crude glycerine}

The aqueous layer was treated with $6 \%$ sulfuric acid to $\mathrm{pH}$ $3-4$. This is to separate the soap and neutralize the remaining sodium hydroxide in the mixture while the charred substances produced during acidification were removed by filtration. The acidified mixture was stabilized for a few minutes to allow settling of aqueous glycerine. The fatty acid found in the upper layer was decanted and stored for reprocessing into a methyl ester. The acidic aqueous glyc- 
erine was added with $50 \%$ sodium hydroxide until the $\mathrm{pH}$ of the solution is 7.0 to neutralize the solution then concentrated via a Buchi rotary vacuum evaporator at $80-90^{\circ} \mathrm{C}$ for the laboratory scale studies. For the scale-up process, a vacuum distillation apparatus, Shibata HP-91000BS was used for the evaporation of water. The operating conditions were: Temperature, $85-105^{\circ} \mathrm{C}$, Pressure, $50-60 \mathrm{~cm} \mathrm{Hg}$. The crystallized salt which was formed during concentration and evaporation was filtered off.

\subsection{Refining of crude glycerine}

The crude glycerine was vacuum distilled with operating conditions: Distillation temperature (top), $120-126^{\circ} \mathrm{C}$; Bottom temperature, $165-190^{\circ} \mathrm{C}$; Pressure, $0.006-0.0006$ psi.

\subsection{Characterization of crude and refined glycerine}

Crude and refined glycerine were analyzed according to the A.O.C.S official method for glycerine (AOCS and Firestone 1989). The samples were analyzed using an RX-1 Perkin Elmer FT-IR spectrophotometer prepared as a thin layer between highly polished $\mathrm{KBr}$ plate.

\section{RESULTS AND DISCUSSION}

Sulfuric acid and sodium hydroxide were used as the reagents for the acidification and neutralization treatment since the aqueous glycerine layer contained a large amount of ash, mainly sodium chloride (Yong et al. 2001) which will form into sodium sulfate, during the reaction. Sodium sulfate is not dissolvable in the hydrous solution of glycerine previously neutralized and drenched with sodium chloride (Helmold 1993; Thomas 1983). Sodium sulfate is easy to form crystals out for easy retrieval by succeeding vaporization and decantation which will reduce the presence of salt in the crude glycerine. The pH during acidification was maintained at 3-4 to reduce the dissolved salt. The formation of salt in the crude glycerine increased at high $\mathrm{pH}$ while at low $\mathrm{pH}$, the salt formation is reduced (Ooi et al. 2001).

Table 1 shows the components of the aqueous layer after chemical treatment (laboratory scale). The average content of crude fatty acids in the aqueous layer is about $32.37 \%(\mathrm{w} / \mathrm{w})$ from a range of $30 \%$ to $35.36 \%$ while the

TABLE 1. The composition of aqueous layer after physical and chemical treatment.

\begin{tabular}{lcc}
\hline & \multicolumn{2}{c}{ Fraction (wt.\%) } \\
\cline { 2 - 3 } & Laboratory scale (1 Kg) & Scale-up (10 Kgs) \\
\hline Crude glycerine & $54.54 \pm 4.51$ & $65.88 \pm 1.96$ \\
Crude fatty acids & $32.37 \pm 2.35$ & $31.80 \pm 2.76$ \\
Salt & $12.47 \pm 4.27$ & $2.05 \pm 1.76$ \\
Charred substances & $0.52 \pm 0.18$ & $0.28 \pm 0.09$ \\
\hline
\end{tabular}

TABLE 2. The physicochemical properties of crude glycerine.

\begin{tabular}{lccc}
\hline & $\begin{array}{c}\text { Laboratory } \\
\text { scale }(1 \mathrm{Kg})\end{array}$ & $\begin{array}{c}\text { Scale-up } \\
(10 \mathrm{Kgs})\end{array}$ & $\begin{array}{c}\text { British Standard } \\
2621: 1979 \text { for crude } \\
\text { glycerine }\end{array}$ \\
\hline $\begin{array}{l}\text { Glycerine } \\
\text { content }(\%)\end{array}$ & $81.20 \pm 0.80$ & $84.92 \pm 4.87$ & 80.0 \\
Water & $8.54 \pm 0.85$ & $4.72 \pm 3.45$ & 10.0 \\
Ash & $8.08 \pm 0.65$ & $8.03 \pm 2.20$ & 2.5 \\
MONG & $2.17 \pm 0.23$ & $2.32 \pm 0.18$ & 10.0 \\
\hline
\end{tabular}

crude glycerine is about $54.54 \%$ from $48.07 \%$ to $58.50 \%$. The salt content is $12.47 \%$. The content $(\mathrm{w} / \mathrm{w} \%)$ of charred substance was determined of about 0.5225 . Scaling-up the process, the average crude glycerin recovered from the aqueous layer was $65.88 \%(\mathrm{w} / \mathrm{w})$ with less formation of salt $(2.05 \%, w / w)$. The results are tabulated in Table 1 . The increase in recovery of crude glycerine showed the viability of the laboratory process for scaling it up.

Other methods of recovering the crude glycerine without the use of a rotary evaporator under vacuum were also employed. Simple heating of the neutralized aqueous glycerine in an open container at low temperature is possible but the glycerine content was only $50 \%-70 \%$ which was below from the $80 \%$ standardized glycerine content for crude glycerine (Sinaga et al. 2019).

The crude glycerine was analyzed for its physicochemical properties and the results are shown in Table 2. The average glycerine content (lab. scale) was $81.20 \%$ and $85.0 \%$ for the scale-up process. The glycerine content from both processes was within the British standard for crude glycerine (Sinaga et al. 2019) as shown in Table 2. The ash content was $8.08 \%$ (lab. scale) and $8.03 \%$ (scale-up process) while the water content was $8.54 \%$ (lab.scale) and $4.72 \%$ (scale-up process). The Matter organic non-glycerol (MONG) of both samples as reflected in Table 2 meets the British standard for crude glycerine (Sinaga et al. 2019)). MONG formation is highly dependent on the $\mathrm{pH}$ during chemical treatment. At low $\mathrm{pH}$, acrolein is formed that further increased the content of MONG (Garti et al. 1981), due to the oxidation of glycerol to glyceraldehyde and dihydroxyacetone.

The recovered crude glycerin was further distilled using a fractional distillation apparatus. The average refined glycerine content is shown in Table 3 . On average, $73.78 \%$ refined glycerine was recovered from the crude glycerine with $10.92 \%$ distilled bottom and $15.30 \%$ distilled water. The reduced content of glycerine in the refined glycerine may be due to three possible reactions. These are: polymerization of the glycerol at high $\mathrm{pH}$ (in excess alkali and high temperature, $>200^{\circ} \mathrm{C}$ ) to form polyglycerol (Jeromin et al. 1998; Matsushita et al. 1990; Sinaga et al. 2019), which accumulates in the distilled bottom; dehydration of the glycerol at low $\mathrm{pH}$ (Hedtke 1996) to form acrolein (bp. $52^{\circ} \mathrm{C}$ ), which

TABLE 3. The composition of distilled fractions recovered from crude glycerine.

\begin{tabular}{lccc}
\hline \multirow{2}{*}{$\begin{array}{l}\text { No. of } \\
\text { Trials }\end{array}$} & \multicolumn{3}{c}{ Fraction \% (w/w) } \\
\cline { 2 - 4 } & Refined glycerine & Distilled bottom & Distilled water \\
\hline 1 & 70.90 & 13.00 & 16.10 \\
2 & 73.40 & 10.32 & 16.28 \\
3 & 77.04 & 9.43 & 13.53 \\
\hline Average & $73.78 \pm 3.10$ & $10.92 \pm 1.86$ & $15.30 \pm 1.54$ \\
\hline
\end{tabular}

TABLE 4. The physicochemical properties of refined glycerine.

\begin{tabular}{lcc}
\hline & $\begin{array}{c}\text { Scale-up } \\
(10 \mathrm{Kgs})\end{array}$ & $\begin{array}{c}\text { USP standard for } \\
\text { refined glycerine }\end{array}$ \\
\hline Glycerol, \% & $96.86 \pm 1.370$ & $95-101$ \\
Water, \% (max) & $1.10 \pm 0.320$ & 5.0 \\
Residue on ignition, \% (max) & $0.009 \pm 0.001$ & 0.01 \\
Refractive Index at $20^{\circ} \mathrm{C}$ & $1.4696 \pm 0.002$ & - \\
Specific gravity, $25^{\circ} \mathrm{C}(\mathrm{min})$ & $1.296 \pm 0.062$ & 1.249 \\
\hline
\end{tabular}


is then lost in the cold trap; formation of glycerose which is a mixture of glyceraldehyde and dihydroxyacetone (Jungermann 1991) due to moderate oxidation of the glycerol. However, these unwanted reactions can be minimized by controlling the $\mathrm{pH}$ of the crude glycerine, including the temperature and pressure of distillation. In this study, fractional distillation under vacuum was used to attain lower pressure and temperature during the recovery of refined glycerine.

The physicochemical properties of the refined glycerine were determined in comparison with the specification set by United States Pharmacopeia for glycerin (USPC 2009) as shown in Table 4. The glycerol content of the refined glycerine is about $97.0 \%$. By fractional distillation under vacuum, the crude glycerine with $81.20 \%$ glycerine content was converted to a higher purity The residue on ignition (ash content) of the distilled glycerine was $0.009 \%$ (Table 4). Fractional distillation also removed the salts (sodium chloride and sodium sulfate), that mainly constituted the ash. Salts have high boiling points because of their strong ionic bonding and classified as inorganic chemicals. Salts are not easy to vaporize and therefore accumulate in the distilled bottom (Yong et al. 2001). The water content in the refined glycerine was $1.10 \%$ (Table 4 ) significantly less than in crude glycerine (8.54\%) (Table 3) due to the application of high vacuum during distillation process that vaporizes the water then condense in the liquid nitrogen cold trap. The use of vacuum and cold trap act as a drying system for the distilled glycerine. The results obtained for the physicochemical properties of refined glycerin (Table 4) were within the specification set by United States Pharmacopeia for glycerin (USPC 2009).

The infrared spectrum of both crude and refined glycerine from the scale-up process is presented in Figure 1. There was O-H stretching at $3294.12 \mathrm{~cm}-1, \mathrm{C}-\mathrm{H}$ stretching at $2879-2929 \mathrm{~cm}-1, \mathrm{C}-\mathrm{O}-\mathrm{H}$ bending at 1417 to $1454 \mathrm{~cm}-1, \mathrm{C}-$ O stretching from 1042 to $1107 \mathrm{~cm}-1$ and $\mathrm{O}-\mathrm{H}$ bending at 922 $\mathrm{cm}-1$. Similar spectra with the commercial refined glycerine (Figure 2) was observed. The IR spectrum of the refined

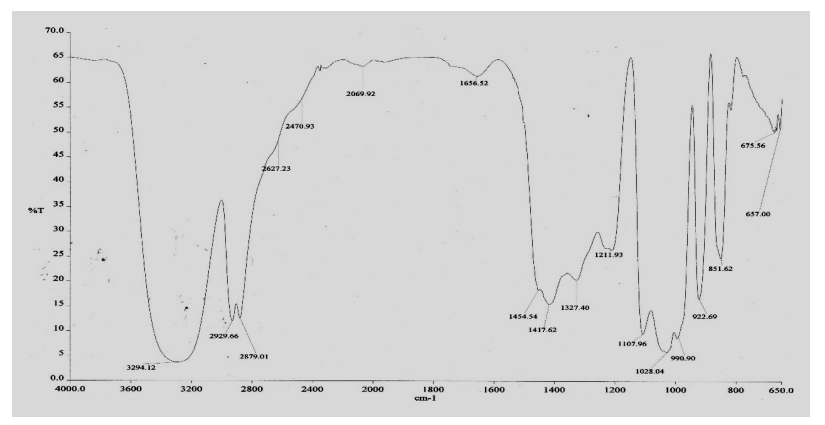

FIGURE 1. The Infrared spectrum of crude glycerine (scale-up process).

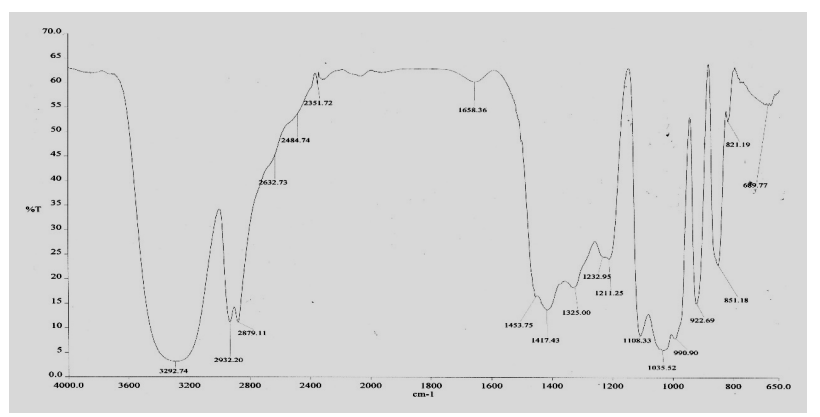

FIGURE 2. The Infrared spectrum of refined glycerine. glycerine is similar to that of commercial glycerin but with a broader absorption band at $3292 \mathrm{~cm}-1$. This might be due to the hydroxyl groups of residual water of the distilled glycerine and the hydroxyl groups of the glycerol molecules.

\section{CONCLUSION}

Crude glycerine can be recovered from the aqueous glycerine layer, a by-product in the production of methyl ester. This is done by subjecting the aqueous glycerine layer with physical and chemical treatment followed by evaporation and concentration. In the chemical treatment of aqueous glycerine layer, three main factors were involved: a. sulfuric acid decreased the solubility of the sodium chloride in the solution, crystallizing it out; b. sodium hydroxide neutralized sulfuric acid to lessen its effect on the solubility of sodium chloride and c. formation of excess salt. Excess sulfuric acid was preferred for the recovery as it reduced salt in the crude glycerine. Fractional distillation under vacuum is a simple and efficient process to refine crude glycerine. Using a $4.0 \times 10-1$ to $4.0 \times 10-2$ mbar pressure at $120-126^{\circ} \mathrm{C}$ produced a glycerine with $96.86 \%$ purity. The optimum $\mathrm{pH}$ for distillation was $<5$ to minimize the formation of foam. This study has established operating parameters that will serve as a guide in the recovery of crude glycerine from the aqueous layer and commercial production of refined glycerine in Philippine setting. The process also showed the technical and economic viability of putting-up a commercial plant for crude and refined glycerine in the Philippines.

\section{ACKNOWLEDGMENTS}

This study was supported by the Industrial Technology Development Institute (ITDI) and the Department of Science and Technology (DOST). Special thanks to the Chemical Process Development Section and Organic Chemicals Section of the Chemicals and Minerals Division of ITDI.

\section{REFERENCES}

[AOCS] American Oil Chemists Society, Firestone D. 1989. Official methods and recommended practices of the American Oil Chemists' Society. 4th edition. Champaign: American Oil Chemists' Society.

Garti N, Aserin A, Zaidman B. 1981. Polyglycerol esters: optimization and techno-economic evaluation. J Amer Oil Chem Soc. 58(9):878-883. doi:10.1007/BF02672963.

Gunstone F, Heming M. 2004. Glycerol-an important product of the oleochemical industry. Lipid Technol. 16(8):177-179.

Hedtke D. 1996. Glycerine processing. In: Hui YH, editor. Industrial oil and fat products. 5th edition. New York: John Wiley \& Sons. p. 275-308.

Helmold VP. 1993. Sodium sulfate. In: Barbara E, editor. Ullmann's encyclopedia of industrial chemistry. 5th edition. Weinheim: VCH Verlagsgesellschaft. p. 355-368.

Jeromin L, Gutsche B, Bunte R, Jordan V, inventors; Cognis Deutschland GmbH and Co KG, assignee. 1998 Jan 20. Process for the production of diglycerol. United States patent US 5,710,350.

Jungermann E. 1991. Chemical reactions of glycerine. In: Jungermann E, Sonntag NOV, editors. Glycerine, a key cosmetic ingredient. New York: Marcel Dekker. p. 97112.

Matsushita K, Naribayashi I, Okuda S. 1990 Jun 29. Production of high-purity diglycerol. Japan patent JPH $02169536 \mathrm{~A}$. 
Ooi TL, Yong KC, Dzulkefly K, Wan Yunus WMZ, Hazimah AH. 2001. Crude glycerine recovery from glycerol residue waste from a palm kernel oil methyl ester plant. J. Oil Palm Res. 13:16-22.

Purdy E, Corpuz PG. 2019. Philippines biofuels annual: Philippine biofuel industry outlook. Technical report. United States Department of Agriculture Foreign Agricultural Service. Manila.

Quispe CA, Coronado CJ, Carvalho JA. 2013. Glycerol: production, consumption, prices, characterization and new trends in combustion. Renewable Sustainable Energy Rev. 27:475-493. doi:10.1016/j.rser.2013.06.017.

Sinaga MS, Ramadhan MR, Rico G, Sitompul WG. 2019. Ef- fect of acidification mole ratio and solvent volume ratio of glycerol purification. IOP Conf Ser: Mater Sci Eng. 505(1):1-5. doi:10.1088/1757-899X/505/1/012116.

Thomas FC. 1983. Sodium sulfate. In: Encyclopedia of chemical technology. 3rd edition. New York: John Wiley \& Sons. p. 245-256.

[USPC] United States Pharmacopeial Convention. 2009. Glycerin. Revision Bulletin. https://www.uspnf.com/ sites/default/files/usp_pdf/EN/USPNF/revisions / 2009-01-30glycerinmonograph.pdf.

Yong KC, Ooi TL, Dzulkefly K, Wanyunus WMZ, Hazimah aH. 2001. Characterization of glycerol residue from a palm kernel oil methyl ester plant. J. Oil Palm Res. 13(2):1-6. 